\title{
Soft Tissue Simulation Based on Measured Data
}

\author{
M. Hauth ${ }^{1}$, J. Gross ${ }^{1,2}$, W. Straßer ${ }^{1}$, and G.F. Buess ${ }^{2}$ \\ 1 WSI/GRIS, University of Tübingen \\ 2 MIC, Department of General Surgery, University Hospital Tübingen
}

\begin{abstract}
Using methods from the computational sciences to improve the quality of health care is an important part of current medical progress. A particulary complex field is surgery simulation, where the fidelity of the systems is still unsatisfactory. We present a finite element system based on a sophisticated material law, better suited for dynamical computations than the standard approaches. To balance computational cost, a hierarchical basis is employed, allowing detail where needed. For time integration the use of a stabilized Runge-Kutta method is proposed.
\end{abstract}

\section{Introduction}

Surgery simulations become an increasingly relevant alternative to traditional training methods for minimally invasive surgeons. Other applications are being envisioned: quantitative, automated assessment of surgical competence, instrument design and testing, biomechanical studies, as well as operation planning and training using patient specific data. Deformable tissue simulations require high computational power; so far this has made simplifications in the models necessary. This leads to limitations in the fidelity of simulated tissue mechanics, hence limiting its usefulness for training beyond simple hand eye coordination. We use concepts borrowed from materials science, geoscience and numerical mathematics to improve on quality of the virtual tissue.

Previous and Related Work: Interactive visual simulations based on physical laws were first investigated in computer graphics, pioneered by Terzopoulos et al. 23. Similarly to most of the newer work the authors used methods from numerical engineering 2611] with the drawback of high computational costs. Early work often lacked the ability to reproduce physical properties. This includes free form deformation models 20, 3D chainmail 8] and mass-spring systems 24, the latter being unable to model incompressibility or transverse contraction without penalty forces. Therefore techniques from numerical engineering, namely finite element and finite difference methods continue to be the most versatile and accurate methods. By now there is sufficient computational power available to allow real-time simulations using physically accurate modelling techniques.

One of the first medical applications was published by Bro-Nielsen [5], who used finite-elements with Cauchy's small strain tensor. Real-time animation based on explicit finite elements using Green's tensor has been presented by Debunne 7]. Capell et al. [6] use a hierarchial basis and linearize around a floating 
reference frame. Hierarchical bases, introduced into numerics during the 80's 3 , have been traced back by Yserentant 25] to the beginning of the 20th century.

Considering time integration methods, more advanced work has been presented in cloth simulation. Since Baraff et al. 4], implicit methods became standard. They allow larger time steps than standard explicit methods, paying the price of solving large linear systems. Our choice for the stabilized explicit timeintegrator is based on stability theory, developed in numerical analysis 1112 .

Linear material laws, although generalized to anisotropic materials[19, are still standard. Measuring stiffness and damping parameters to make the simulation more realistic has been addressed by Maaß 16] and Szekely 22] with various methods. Neither of these groups implemented their viscoelastic model into the simulation. The measurement techniques are currently refined by Nava et al. 17. Recently, Ottensmeyer et al. 14] was able to determine viscoelastic tissue properties over a reasonably wide frequency range. He compared his indentation method and the rotary shear method developed in our group. The results still show some discrepancies between the methods, but confirm the constant $Q$ model as applicable for tissue in vivo. In the future, non-invasive methods such as elastography based on NMR imaging 21] are most promising.

\section{The Physical Model}

Mathematically a deformable solid can be described by it rest state $\Omega$ and a family of displacement fields $u_{t}: \mathbb{R}^{3} \supset \Omega \rightarrow \mathbb{R}^{3}$. The position of a point $x$ in space at time $t$ is then given by $x+u_{t}(x)$. From continuum mechanics [2], we know that the arising elastic energy is given by the outer tensor product

$$
E_{e l}=\int_{\Omega} \sum_{i, j} \epsilon_{i j} \sigma_{i j}=: \int_{\Omega} \epsilon: \sigma
$$

with the symmetric strain tensor $\epsilon$ and the symmetric stress tensor $\sigma$. Stress and strain are related by a material law, mapping the current strain to the current stress

$$
\sigma=C(\epsilon)
$$

which can be substituted into (2.1). All that remains is to decide about how to compute $\epsilon$ and what kind of material law to apply.

Elastic Strain Tensors: There are two common strain tensors for Lagrangian formulations, the linear Cauchy strain tensor $\epsilon^{C}$ and its nonlinear generalization, Green's tensor $\epsilon^{G}$,

$$
\epsilon_{i j}^{C}=\frac{1}{2}\left(\frac{\partial u_{i}}{\partial x_{j}}+\frac{\partial u_{j}}{\partial x_{i}}\right), \quad \text { and } \quad \epsilon_{i j}^{G}=\frac{1}{2}\left(\frac{\partial u_{i}}{\partial x_{j}}+\frac{\partial u_{j}}{\partial x_{i}}+\sum_{k} \frac{\partial u_{k}}{\partial x_{i}} \frac{\partial u_{k}}{\partial x_{j}}\right) .
$$

Using Cauchy's tensor is computationally much more efficient [5], as function evaluations are cheaper and the system remains linear, but is has the serious drawback that rigid body movements produce artificial forces which leads to distortions [18]. Therefore Green's tensor will be used, denoted as $\epsilon$. 


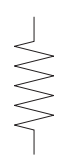

(a)

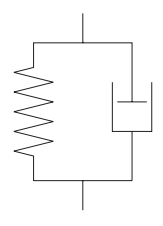

(b)

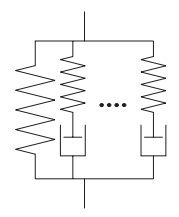

(c)

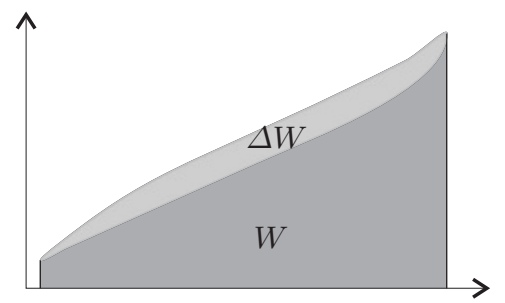

Fig. 1. Linear material laws ((a) Hooke, (b) Kelvin-Voigt, (c) Prony).

Fig. 2. Hysteresis and mechanical Quality

Material Laws: Hooke's law assumes a linear relation between the two tensors

$$
\sigma=C \epsilon,
$$

and is almost exclusively used in current simulators. For isotropic materials only two of the 36 entries of $C$ are distinct, the Lamé constants $\lambda$ and $\mu$. In an anisotropic setting more constants remain 19 .

In $1 \mathrm{D}$ this reduces to a spring $f=k d u$ (Fig. 1(a)). For a dynamical simulation including inertia, we need viscous damping, usually added by replacing the strain tensor $\epsilon$ in (2.2) by the strain rate tensor $\dot{\epsilon}$. Again in 1D this is a spring-damper element (fig. 1(b)).

In dynamical simulations, time dependent phenomena come into play. Most remarkable are stress relaxation and creep, i.e. the material becomes weaker under continual stress and, when unloaded, creeps slowly back to its rest state. For example when a piece of tissue is pulled with a forceps for a while and then released, it will not return immediately but slowly creep back. The KelvinVoigt/Hooke model has a simple exponential relaxation and creep law, which is usually not sufficient to reproduce this behavior accurately.

The most general material law to describe a visco-elastic response is given by the convolution with the unit-step response

$$
\sigma(t)=\int_{-\infty}^{t} \hat{G}(t-\tau) \dot{\epsilon}(\tau) d \tau .
$$

Without any restriction to the material relaxation function $\hat{G}$, this describes any linear visco-elastic material. As it is practically impossible to store the complete history of strain, one usually develops $\hat{G}$ into an exponential Prony series

$$
\hat{G}(t) \approx G(t):=\mu_{0}+\sum_{i=0}^{m} \mu_{i} e^{-t / \lambda_{i}} .
$$

The usefulness of the series expansion becomes clear, when inserting (2.6) into (2.5). Integration yields

$$
\sigma(t)=\mu_{0} \epsilon(t)+\sum_{i=0}^{m} \mu_{i}\left[e^{\frac{t-t_{0}}{\lambda_{i}}} \int_{-\infty}^{t_{0}} e^{-\frac{t_{0}-\tau}{\lambda_{i}}} \dot{\epsilon}(\tau) d \tau+\int_{t_{0}}^{t} e^{-\frac{t-\tau}{\lambda_{i}}} \dot{\epsilon}(\tau) d \tau\right]
$$




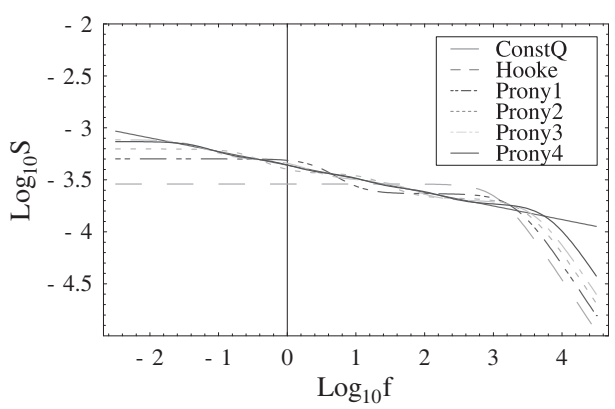

(a) Compliance $S$

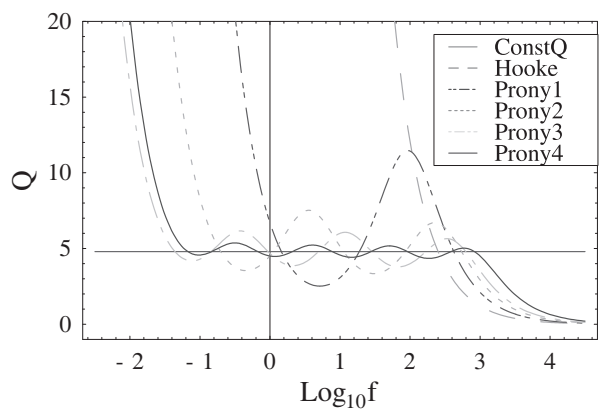

(b) Mechanical Quality Q

Fig. 3. Constant Q fits in the frequency domain.

for any $t_{0}<t$. This is almost perfect for a numerical integration scheme, where we know the strain of the last time step $t_{n}$. Approximating the strain rate tensor $\dot{\epsilon}$ linearly during a time step gives an update scheme

$$
\begin{aligned}
q_{i}(t) & =e^{\Delta t / \lambda_{i}} q_{i}\left(t_{n}\right)+\Delta q_{i}, \text { with } \Delta q_{i}=\left(\lambda_{i} / \Delta t\right)\left(1-e^{\Delta t / \lambda_{i}}\right)\left(\epsilon(t)-\epsilon\left(t_{n}\right)\right) \\
\sigma(t) & =\mu_{0} \epsilon(t)+\sum_{i=0}^{m} \mu_{i} q_{i}(t),
\end{aligned}
$$

where $\Delta t=t-t_{n}, t>t_{n}$. The memory parameter array $q_{i}$ needs to be updated when advancing a time step and represents the history component of the strain.

Mechanical Quality: Now that we are able to simulate practically any linear material, we need to make a choice for a model for soft tissue. Fung[9] states that 'in tissues the shape of the hysteresis loop is invariant over many orders of magnitude in frequency'. Newer ex- and in-vivo measurements confirm this 10.

A measurement for hysteresis is the mechanical quality factor $Q$ :

$$
Q:=\frac{4 \pi W}{\Delta W},
$$

with $W$ being the average elastic energy during a hysteresis loop and $\Delta W$ the dissipated energy (fig. 22). Consequently a material with $Q=0$ absorbs all energy, like a fluid, and a high $Q$ material possesses almost no hysteresis, like a steel spring. The function $\hat{G}$ of a material with constant $Q$ is complicated 15 . With $\gamma=1 / \tan (\pi Q)$ and $M_{0}$ being a reference modulus at $t_{0}$ we have

$$
\hat{G}(t)=\frac{M_{0}}{\Gamma(1-2 \gamma)}\left(\frac{t}{t_{0}}\right)^{-2 \gamma} .
$$

To simulate this material, we fit the parameter $\lambda_{i}, \mu_{i}$ of a Prony series element to the relaxation function. Instead of directly performing the nonlinear 
fit on 2.10) we rather match the compliance functions $S$ of both models, minimizing the relative $L_{2}$-error. This eases the numerical part of the fit and gives a good reproduction of relaxation and creep experiments. Note that this fitting process is exactly the one we described previously [10, as the Prony series in 1 -space corresponds to the relaxation function of a mass spring-network1. A last observation eases this fit. The phenomena were are interested in in visual simulation are in the range of human perception, which we rate between $10 \mathrm{kHz}$ (haptics) to $0.1 \mathrm{~Hz}$. Therefore it suffices to fit constant Q behavior in this range. More details can be found in 10]. Figure 3 shows fits with 2 to 5 memory parameters. For comparison we added a single $\mathrm{KV}$-element. Its mechanical quality $Q(\omega)=k /(\omega d)$ is strongly frequency dependent.

\section{Space and Time Discretisation}

Due to space restrictions we only briefly describe the transformation to an ordinary differential equation using finite elements and refer to Hauth et al. [13.

By minimizing the energy given in (2.1), i.e. equating its first variation to 0 , adding body forces $b(x)$, surface traction $t(x)$, inertia and viscous damping forces, we arrive at the weak formulation of the partial differential equation

$$
\int_{\Omega} \delta u \rho \ddot{u} d x+\int_{\Omega} \delta \epsilon: C(\epsilon) d x+\int_{\Omega} \delta \dot{\epsilon}: D(\dot{\epsilon}) d x-\int_{\Omega} \delta u b d x-\int_{\delta \Omega} \delta u t d s=0 .
$$

As in a standard fine element approach $u$ and $\delta u$ are replaced by piecewise linear approximations over a tetrahedral decomposition of $\Omega$. Inserting this approximation into the principle of virtual work (3.1) leaves an ODE. All integrals are evaluated piecewise over the elements, and summed up. Arranging the computations carefully gives a cost of around 600 flops per element for the evaluation of the elastic and viscous forces with a Hooke law. Each memory parameter adds about 20 flops per element, thus the impact per memory parameter is less than $5 \%$. We apply row sum mass lumping and construct a series of nested meshes by octasection of each tetrahedron. Using the efficient $\mathrm{O}(\mathrm{N})$ wavelet transformation $S$ [25], is it possible to carry out all computations using the wavelet approximation of $u$ with minimal changes to the code. Based on the hierarchical basis $u$ can be easily approximated adaptively. To choose, where to refine, we use a criterion similar to Debunne et al.[7] and decide upon the internal shear forces. We complete the model by static and dynamic surface friction forces.

A detailed analysis of the ODE reveals, that the problem is stiff, which has important consequences for time integration. Using an explicit integration methods, the admissible time step for the numerical solution to be stable is often severely limited. This restriction is less severe for stabilized methods, which are better suited than the classical Runge-Kutta methods like RK4, DOPRI or Cash/Karp. The Orthogonal-Runge-Kutta-Chebychev methods (ROCK) [1],

\footnotetext{
${ }^{1}$ Though a continuous material, given by a Prony series law and discretized by finite elements, is not equivalent to a mass-spring network.
} 
Table 1. Runtimes on a P4-2000.

\begin{tabular}{c|l||r|r} 
Cube & Material & time & av. step \\
\hline small & Hooke & $7.4 \mathrm{~s}$ & $\approx 1.5 \mathrm{~ms}$ \\
& constQ & $10.7 \mathrm{~s}$ & $\approx 1.4 \mathrm{~ms}$ \\
\hline large & Hooke & $2.2 \mathrm{~s}$ & $\approx 7 \mathrm{~ms}$ \\
& Hooke2 & 2.4 & $\approx 6 \mathrm{~ms}$ \\
constQ & 2.6 & $\approx 7 \mathrm{~ms}$
\end{tabular}

(a) $8 \mathrm{~s}, \mathrm{ROCK} 2$.

\begin{tabular}{l||r|r|r} 
solver & time & av. step & stages \\
\hline ROCK2 & $2.6 \mathrm{~s}$ & $\approx 7 \mathrm{~ms}$ & $\approx 4$ \\
DOPRI853 & $13.4 \mathrm{~s}$ & $\approx 9 \mathrm{~ms}$ & $\approx 8$ \\
DOPRI5 & 28.7 & $\approx 3 \mathrm{~ms}$ & 5 \\
Midpoint & 6.0 & $1.5 \mathrm{~ms}$ & 2
\end{tabular}

(b) 8s, large cube with constQ.

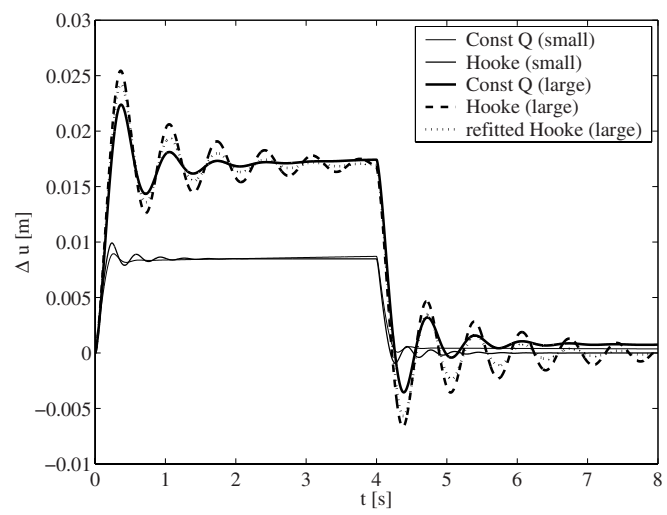

Fig. 4. Ex.1: Displacement of the upper right corner.

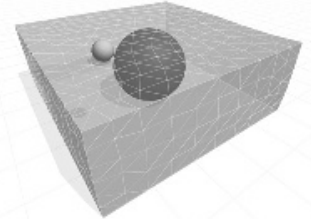

Fig. 5. Ex.2: Embedded object.
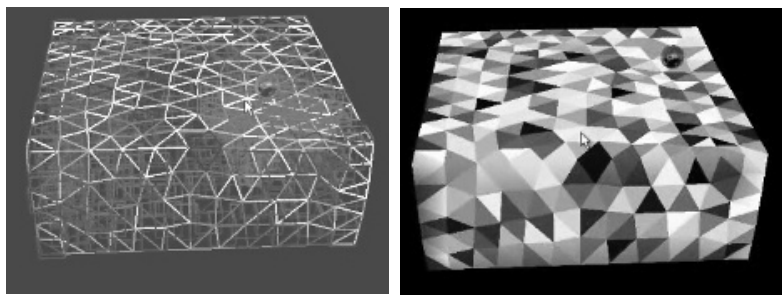

Fig. 6. Ex.2: 431/3448 Tetrahedra (coarse/fine). Wireframe/Wavelet hierarchy and rendered surface.

which we employ, are a new family of methods with a large stability domain, allowing time steps usually about 2 to 10 times larger than classical methods.

\section{Results and Conclusion}

Our first example will show the advantages of using the constant $\mathrm{Q}$ material. A shear force with a force density of $100 \mathrm{~N} / \mathrm{m}^{2}$ is applied to the top surface 

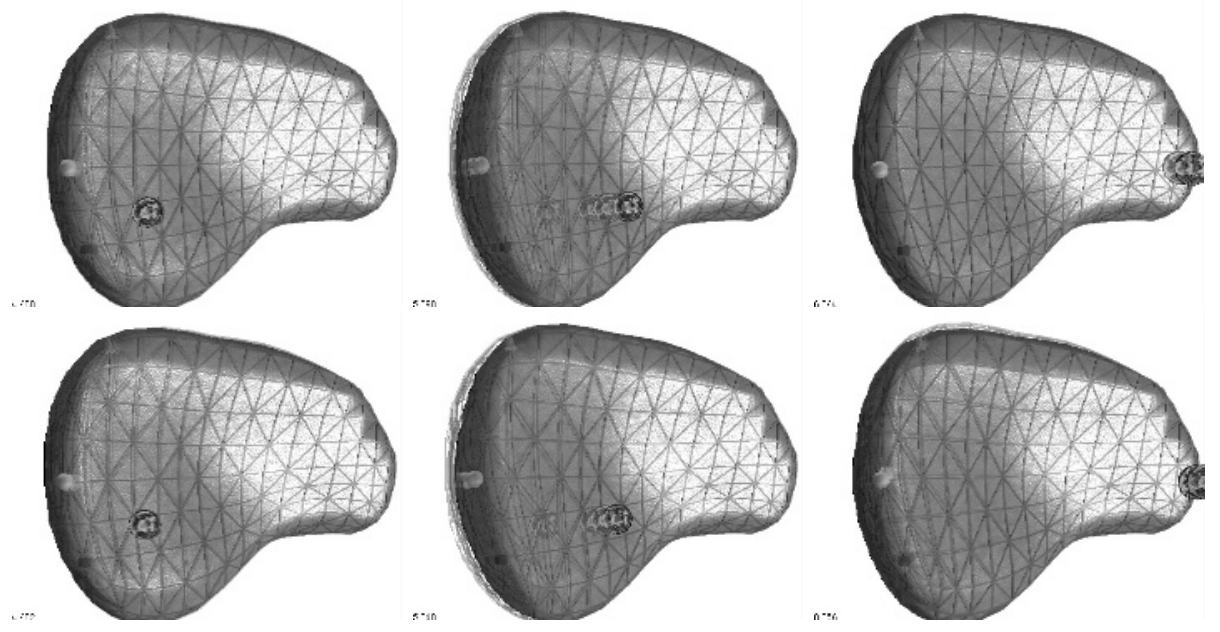

Fig. 7. Ex.3: Liver with 327/2616 Tetrahedra (coarse/fine). Three snapshots with a constant $\mathrm{Q}$ material (upper row), followed by a Hooke material (lower row). The time is given in the corner. Video provided at http://www.gris.unituebingen.de/projects/virtis/miccai.html

of a cube with $0.1 \mathrm{~m}$ edge length, which is composed of 327 tetrahedra. The bottom of the cube is fixed to the ground. We apply the force for $4 \mathrm{~s}$ and let the cube swing to rest afterwards. The red cube has a constant $\mathrm{Q}$ material ( $Q=4.8$ and $\mu=2.5 \mathrm{kPa} @ 2 \mathrm{~Hz}$, measured for liver tissue[10]), fitted with 3 memory parameters, the blue one has a Hooke material. As the liver is filled with blood, we choose a Poisson ratio of 0.49995 . The constants $\lambda$ and $\mu$ of the Hooke material (blue cube) are fitted, such that it possesses a $Q$ of 4.8 at the resonance frequency of the cube. It oscillates longer and does not show the correct creep behavior (fig. 4). In the second part, we rescaled both cubes with a factor of two, keeping all material constants. Now the blue cube gives a different behavior and oscillates much longer. This is due to its new resonance frequency. The new green cube is again a Hooke material with a model fitted to the new resonance frequency. Thus using Hooke's law, one has to tune the parameters to the given geometry which is not always as easy as in the case of a cube.

To show the efficiency of ROCK, we redid the last simulation with other explicit solvers. DOPRI5 and DOPRI853 were taken from Hairer et. al. 11, the explicit midpoint rule is a customized implementation using a constant time step. All run times are given in table 1. As predicted, the performance impact of the memory parameter model is about $15-20 \%$.

The second experiment employs a cube of $25 \times 25 \times 5 \mathrm{~cm}$, with an embedded region with different material properties. The material inside the blue sphere (fig. 5) has the same constant Q material as above. The outside has a lower $\mu=$ $0.4 k P a$ and a Poisson ratio of 0.3 . For the simulation we use a two-level wavelet basis. The tetrahedra of the higher level are darker, the lower levels are activated when the pressure of the tool gives rise to high forces. The snapshots (fig. 6) show, how the material reacts different. Using haptic feedback, one can feel the 
embedded object. We provide a video of this as well as of all other experiments at www.gris.uni-tuebingen.de/projects/virtis, live captured from the application. The ratio between real and simulation time was between 0.4 and 0.7 .

The last experiment employs a liver model (about 20x30x20 cm) (fig. 7), fixed at the right back (from viewpoint). The first part of the animation is again performed using the constant Q material from above. The second one uses the Hooke material, fitted to the larger cube. We tried to illustrate the quite different dynamical effects by ghosting and attaching markers in fig. 7 Note that the Hooke material oscillates much longer. The subjective impression of the video is even more drastic. It can again be found at the above location. The frame rates are again about $60-75 \%$ real-time.

Conclusion: In this paper we presented a new framework for the simulation of soft tissue. Our approach combines three techniques. First, we have presented a new material model. It is the first implementation of a real dynamic material, incorporating a defined frequency behavior, that is capable of modelling organic materials accurately. Second, the implementation is based on a simple wavelet basis. By this, adaptivity is effortlessly added, irregular refinements need not to be handled. Therefore detail can be added where needed, allowing an accurate and fast simulation without being forced to perform a costly global refinement. Third, we introduced a stable integration method, that combines the advantages of explicit methods with the enhanced stability of implicit methods.

\section{References}

1. A. Abdulle and A. A. Medovikov. Second order Chebyshev methods based on orthogonal polynomials. Numer. Math., 90(1): 1-18, 2001.

2. T. M. Atanackovic and A. Guran. Theory of Elasticity for Scientists and Engineers. Birkhäuser, Boston, 2000.

3. R. E. Bank. Hierarchical bases and the finite element method. Acta Numerica, 5: 1-43, 1996.

4. D. Baraff and A. Witkin. Large steps in cloth simulation. In M. Cohen, editor, SIGGRAPH 98 Conference Proceedings, pages 43-54, July 1998.

5. M. Bro-Nielsen. Finite Element Modeling in Medical VR. Journal of the IEEE, 86(3): 490-503, 1998.

6. S. Capell, S. Green, B. Curless, T. Duchamp, and Z. Popovic. Interactive skeletondriven dynamic deformations. In SIGGRAPH 2002, pages 586-593. ACM SIGGRAPH, 2002.

7. G. Debunne, M. Desbrun, M.-P. Cani, and A. H. Barr. Dynamic Real-Time Deformations using Space and Time Adaptive Sampling. In SIGGRAPH 2001, pages 31-36, 2001.

8. S. F. Frisken-Gibson. Using Linked Volumes to Model Object Collisions, Deformation, Cutting, Carving, and Joining. IEEE TVCG, 5(4): 333-348, Oct. 1999.

9. Y. C. Fung. Biomechanics: Mechanical properties of living tissues. Springer, New York, 1993.

10. J. Groß, M. Hauth, O. Etzmuß, and G. F. Buess. Modelling Viscoelasticity in Soft Tissues. In Int. Workshop on Deformable Modelling and Soft Tissue Simulation. Elsevier, Nov. 2001. 
11. E. Hairer and G. Wanner. Solving Ordinary Differential Equations II. SpringerVerlag, Berlin, 1996.

12. M. Hauth and O. Etzmuß. A High Performance Solver for the Animation of Deformable Objects using Advanced Numerical Methods. In Proc. Eurographics, pages 137-151, Manchester, UK, 2001.

13. M. Hauth, J. Gross, and W. Straßer. Interactive physically based solid dynamics. In Proc. SIGGRAPH Symposium on Computer Animation 2003. ACM Press, 2003.

14. D. Kalanovic, M. P. Ottensmeyer, J. Groß, and S. L. Dawson. Independent testing of soft tissue visco-elasticity using indentation and rotary shear deformations. In Proc. MMVR, 2003.

15. E. Kjartansson. Constant-Q, Wave propagation and attenuation. Journal of Geophysical Research, 85: 4737-4748, 1979.

16. H. Maaß and U. Kühnapfel. Noninvasive measurement of elastic properties of living tissue. In EMBEC'99, volume 37, pages 1460-1461, 1999.

17. A. Nava, E. Mazza, F. Kleinermann, N. Avis, and J. McClure. Determination of the mechanical properties of soft human tissues through aspiration experiments. In MICCAI 2003, 2003.

18. G. Picinbono, H. Delingette, and N. Ayache. Real-Time Large Displacement Elasticity for Surgery Simulation: Non-Linear Tensor-Mass Model. In MICCAI 2000, pages 643-652, October 2000.

19. G. Picinbono, H. Delingette, and N. Ayache. Non-linear and anisotropic elastic soft tissue models for medical simulation. In ICRA2001, May 2001.

20. T. W. Sederberg and S. R. Parry. Free-Form Deformation of Solid Geometric Models. SIGGRAPH '86 Proceedings, 20(4): 151-160, Aug. 1986.

21. R. Sinkus, J. Lorentzen, D. Schrader, M. Lorenzen, M. Dargatz, and D. Holz. High-resolution tensor MR elastography for breast tumour detection. Physics in Medicine and Biology, 45: 1649-64, 2000.

22. G. Szekely, C. Brechbühler, R. Hutter, and A. Rhomberg. Modelling of soft tissue deformation for laparoscopic surgery simulation. In Proc. MICCAI98, 1998.

23. D. Terzopoulos and K. Fleischer. Deformable Models. The Visual Computer, 4: 306-331, 1988.

24. A. Van Gelder. Approximate Simulation of Elastic Membranes by Triangle Meshes. Journal of graphics tools, 3: 21-42, 1998.

25. H. Yserentant. Hierarchical bases. In ICIAM91, pages 281-290. SIAM, 1992.

26. O. Zienkiewicz and R. Taylor. The finite element method. Vol. 1-3. Oxford: Butterworth-Heinemann., 2000. 